\title{
Fluorine Abundance Variations in M4
}

\author{
Verne V. Smith ${ }^{1}$ \\ ${ }^{1}$ National Optical Astronomy Observatory, Tucson, USA \\ email:vsmith@noao.edu
}

\begin{abstract}
Abundance variations in globular clusters have been observed for the light elements $\mathrm{C}, \mathrm{N}, \mathrm{O}, \mathrm{Na}, \mathrm{Al}$, as well as sometimes $\mathrm{Mg}$. Such variations can be understood as the result of H-burning. In particular for the globular cluster $\mathrm{M} 4$, anticorrelations of $\mathrm{O}$ with $\mathrm{Na}$ and $\mathrm{Al}$ have been established from previous studies. In this study, we discuss fluorine abundances in a sample of M4 red-giants derived from infrared high-resolution spectra obtained with Pheonix on Gemini South. Our results show that fluorine now can be added to the suite of elements that are shown to vary in M4: the abundance of ${ }^{19} \mathrm{~F}$ is found to be anti-correlated with the sodium and aluminium variations. These results provide further evidence that H-burning is indeed responsible for the observed chemical inhomegeneities. Moreover, from comparisons with stellar models, tighter constraints on the mass of the polluting star in this globular cluster are extablished.
\end{abstract}

Keywords. Stars: abundances, globular clusters: individual (M4)

\section{Introduction}

Galactic globular clusters have abundance patterns that are distinct from those of the field and show variations in the abundances of the light elements $\mathrm{C}, \mathrm{N}, \mathrm{O}, \mathrm{Na}, \mathrm{Al}$ and $\mathrm{Mg}$. The sense of these variations is such that the abundances of $\mathrm{C}$ and $\mathrm{N}, \mathrm{O}$ and $\mathrm{Na}$, and $\mathrm{Al}$ and $\mathrm{Mg}$ are anti-correlated. These abundance variations in globular clusters can be understood to result from H-burning (or proton captures) reactions involved in the CNO, $\mathrm{Ne}-\mathrm{Na}$ and $\mathrm{Mg}-\mathrm{Al}$ cycles. For an extensive review on the subject we refer to Gratton, Sneden, \& Carretta (2004).

Globular cluster abundance variations have been mapped typically in giants due to their brightness. Red giants themselves have convective envelopes that mix material to their surfaces that has experienced some H-burning; $\mathrm{C}$ and $\mathrm{N}$ abundance anti-correlations are predicted and observed (e.g. Charbonnel et al. 1998; Gratton et al. 2000). However, variations in $\mathrm{O}, \mathrm{Na}, \mathrm{Mg}$, or $\mathrm{Al}$ in low-mass giants need significantly deeper mixing of material (not predicted by models of low-mass stars). As a solution, extra-mixing scenarios have been constructed for low-mass giants that can explain the globular clusters abundance pattern (e.g. Langer \& Hoffman 1995). On the other hand, nagging observations of anti-correlated $\mathrm{C}$ and $\mathrm{N}$ abundances in turn-off and main-sequence globular cluster stars (Suntzeff \& Smith 1991), plus N and Na correlations in turn-off stars (Briley et al. 1996) cannot be explained by in situ deep mixing. Moreover, recent high-resolution studies (e. g. Ramirez et al. 2001; Gratton et al. 2001, Ramirez \& Cohen 2003) found the same abundance variations in stars close to the main sequence as those observed in the red-giant stars. The $\mathrm{CNO}$ and $\mathrm{Na}-\mathrm{Mg}-\mathrm{Al}$ abundance variations now observed in globular cluster turn-off stars suggest that a site other than the presently observed red giants is needed to produce the hot H-burning abundance patterns specific to the globular clusters.

Massive asymptotic giant branch (AGB) stars are an alternative site where H-burning, mixing, and mass loss take place and that could, in principle, be responsible for the contamination of the early globular cluster environment. The base of the deep and convective 
envelopes of massive AGB stars undergo hot H-burning, or, hot bottom burning (HBB); a process that could explain the observed $\mathrm{CNO}$ and $\mathrm{Na}-\mathrm{Mg}-\mathrm{Al}$ abundance patterns in globular clusters. It is interesting to note that this same process would destroy fluorine via ${ }^{19} \mathrm{~F}(\mathrm{p}, \alpha){ }^{16} \mathrm{O}$ and that ${ }^{19} \mathrm{~F}$, on the other hand, is produced in AGB stars of lower mass (Jorissen et al. 1992); although it is destroyed in HBB occuring in stars with masses above $3.5 \mathrm{M}_{\odot}$.

Fluorine abundances can be derived from HF lines near $2.2 \mu \mathrm{m}$. Here we discuss the abundance patterns in the globular cluster M4 (from Ivans et al. 1999), and add ${ }^{19} \mathrm{~F}$ abundances. The idea being that if massive AGB stars polluted the cluster medium while star formation was still going on (via slow winds from HBB envelopes) the O, Na, $\mathrm{Mg}$, and $\mathrm{Al}$ abundance variations might be explained. The ${ }^{19} \mathrm{~F}$ would then be expected to be significantly depleted.

\section{Observations \& Analysis}

A sample of 7 red-giants were observed with the Phoenix spectrograph (Hinkle et al. 2002) on the Gemini South telescope. The target stars were selected from Ivans et al. (1999). The observed spectra have a resolution $\mathrm{R}=\lambda / \Delta \lambda=50,000$ (corresponding to a resolution element of $\sim 4$-pixels). The spectra were centered in order to contain the molecular $\mathrm{HF}(1-0)$ R9 line near $\sim 23,400 \AA$.

The critical parameters effective temperature $\left(\mathrm{T}_{\text {eff }}\right), \log \mathrm{g}$, microturbulent velocity $(\xi)$, and metallicity for the target stars were taken from the Ivans et al. study. The effective temperatures were based upon line-depth ratios, and the surface gravities were derived from the ionization equilibrium of both Fe I/Fe II, and Ti I/Ti II. The microturbulent velocities were obtained from the sample Fe I lines, which covered a significant range in equivalent width.

Fluorine abundances were obtained from calculations of synthetic spectra for the spectral region containing the HF (1-0) R9 line. For details of the adopted linelist we refer to Smith et al. (2002) and Cunha et al. (2003).

\section{Discussion}

Figure 1 displays the abundance patterns observed for several elements in the globular cluster M4. The abundances of $\mathrm{O}, \mathrm{F}, \mathrm{Mg}, \mathrm{Al}$, Ti and La are plotted versus sodium, which is selected as a comparison element. Since we are searching for primordial signatures, we do not add the elements $\mathrm{C}$ and $\mathrm{N}$ to our discussion, as these elements are altered by mixing in low-mass red-giants. It is clear, as previously known, that oxygen decreases as the sodium abundance increases; $\mathrm{Al}$ and $\mathrm{Na}$ are positively correlated as well. The novelty here is the addition of the fluorine behavior in this scenario: it can be seen that the behavior of fluorine with sodium is similar to that of oxygen with sodium; or, the fluorine abundances decrease as $\mathrm{Na}$ increases, with a larger variation than oxygen. The other elements do not show any significant variations, within the expected uncertainties. We note that the fact that La does not vary indicates no significant contribution from the s-process.

The results obtained for ${ }^{19} \mathrm{~F}$ displayed in Figure 1 indicate that fluorine can now be added to the suite of elements found to vary in the globular cluster M4. The addition of this element is important because it helps to constrain the models in terms of the mass of the polluting stars. The signature of H-burning from hot-bottom burning in massive AGB stars remains a viable candidate when ${ }^{19} \mathrm{~F}$ depletion is included. Some mass range of AGB stars in an early generation of M4 stars presumably added ejecta to the M4 

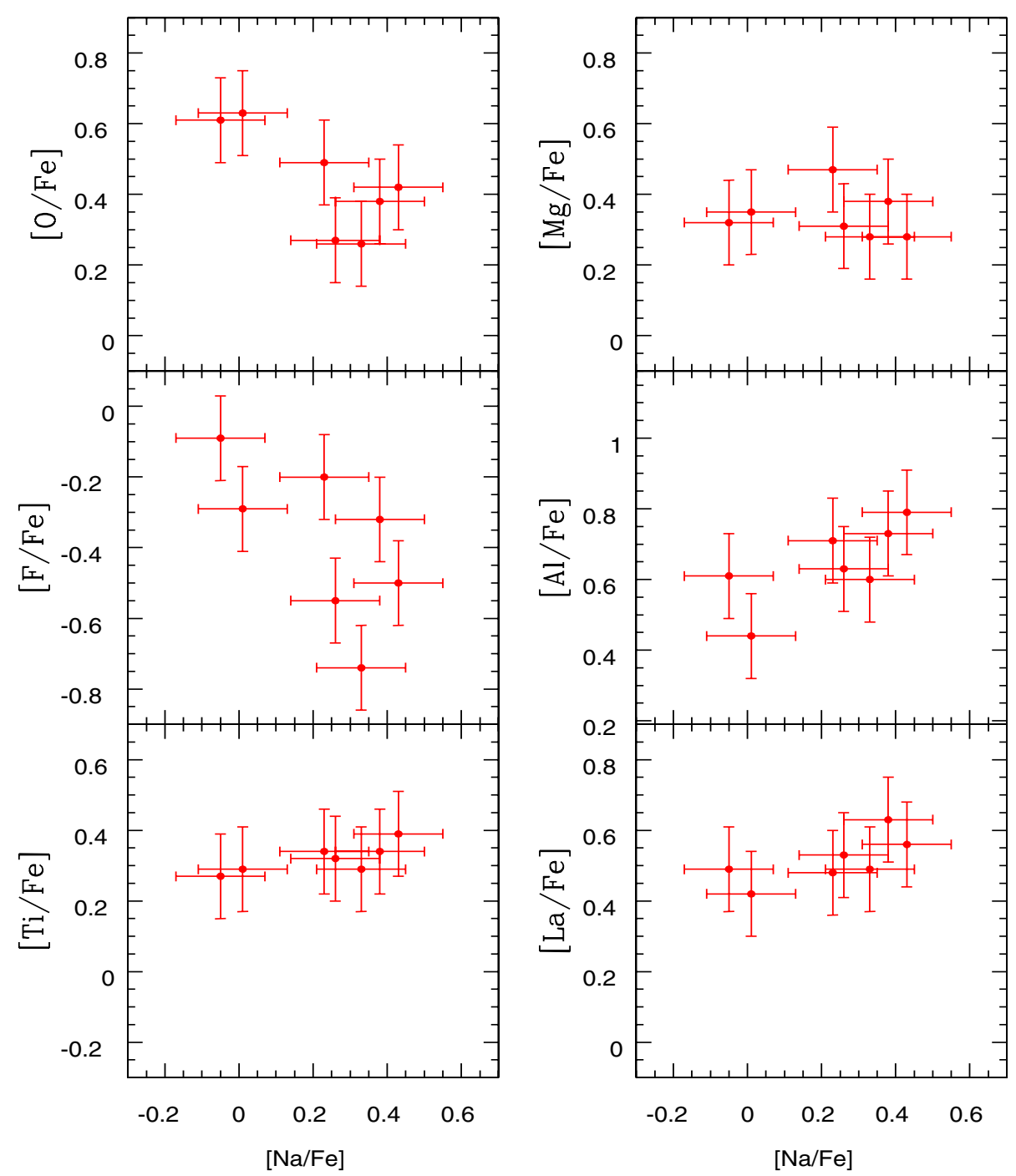

Figure 1. The abundances of oxygen, fluorine, titanium (left panels) magnesium, aluminium and lanthanum (right panels) relative to Fe versus the sodium to iron abundance ratios for our sample of M4 stars. Note the similar behaviors of $[\mathrm{F} / \mathrm{Fe}]$ and $[\mathrm{O} / \mathrm{Fe}]$ that show anti-correlations with $[\mathrm{Na} / \mathrm{Fe}]$, while $[\mathrm{Al} / \mathrm{Fe}]$ increases with $[\mathrm{Na} / \mathrm{Fe}]$.

intracluster environment bearing the signature of their hydrogen burning as revealed by the $\mathrm{C}, \mathrm{N}, \mathrm{O}, \mathrm{Na}, \mathrm{Al}$, and $\mathrm{F}$ abundance variations. This material did not originate in stars more massive than about $8-10 \mathrm{M}_{\odot}$, as there is no evidence of significant variations in those elements produced in SN II. It is a possibility that the high velocity SN II eject might have escaped the intra-cluster medium.

An important step in the understanding of the chemical history of any system is to constrain the masses of its polluting stars. A simple model for the globular cluster M4 can be put together by considering a new generation (or generations) of stars which were 
formed from different fractions of stellar ejecta, thus resulting in the observed abundance variations and inhomogeneities. What is of special importance here is the observed large depletion of fluorine. Based on models by Fenner et al. (2004) stars with masses less than about 3.5 solar masses are net producers of fluorine during AGB evolution. Our results rule out stars of this mass, or less, as being significant contributors to chemical evolution within M4.

\section{Conclusions}

We discuss the fluorine abundance pattern observed in the globular cluster M4. The main observational result is that the fluorine abundance varies in this globular cluster. The observed fluorine abundance variations correlate with oxygen and anti-correlate with the $\mathrm{Na}$ and $\mathrm{Al}$ variations. The addition of fluorine to the suite of elements that are know to vary in M4 can be used as a significant constraint in defining the masses of the primordial stars which are responsible for the initial pollution in M4.

\section{References}

Briley, M.M., Smith, V.V., Suntzeff, N.B., \& Lambert, D.L. 1996, Nature, 383, 604

Charbonnel, C., Brown, J.A., \& Wallerstein, G. 1998, A \& A, 332, 204

Cunha, K., Smith, V.V., Lambert, D.L. \& Hinkle, K.H., 2003, ApJ, 126, 1305

Fenner, Y., Campbell, S., Karakas, A.I., Lattanzio, J.C., \& Gibson, B.K., 2004, MNRAS, 353, 789

Gray, D. F. 1994, PASP, 106, 1248

Gratton, R., Sneden, C., \& Carretta, E. 2004, ARAA, 42, 385

Gratton, R., and 16 co-authors 2001, A\&A, 369, 87

Gratton, R., Sneden, C., Carretta, E., \& Bragaglia, A. 2000, A\&A, 354, 169

Gustafsson, B., Bell, R.A., Eriksson, K., \& Nordlund, A. 1975, 42, 407

Hinkle, K.H., Blum, R., Joyce, R.R., Ridgway, S.T., Rodgers, B., Sharp, N., Smith, V., Valenti, J., \& van der Bliek, N. 2002, SPIE, 4834, 353

Ivans, I.I., Sneden, C., Kraft, R.P., Suntzeff, N.B., Smith, V.V., Langer, G.E., \& Fulbright, J.P. 1999, 118, 1273

Jorissen, A., Smith, V.V., \& Lambert, D.L. 1992, A\&A, 261, 164

Langer, G. E., \& Hoffman, R. D. 1995, PASP, 107, 1177

Lee, S.W. 1977, A\&AS, 27, 367

Ramirez, S.V., Cohen, J.G., Buss, J., \& Briley, M.M. 2001, AJ, 122, 1429

Ramirez, S.V., \& Cohen, J.G. 2003, AJ, 125, 224

Smith, V.V., Hinkle, K.H., Cunha, K., Plez, B., Lambert, D.L., Pilachowski, C.A., Barbuy, B., Melendez, J., Balachandran, S., Bessel, M.S., Geisler, D.P., Hesser, J.E., \& Winge, C. 2002, AJ, 124, 3241

Suntzeff, N.B., \& Smith, V.V. 1991, ApJ, 381, 160 